\title{
De Novo Assembly of the Transcriptome of Turritopsis, a Jellyfish that Repeatedly Rejuvenates
}

\section{$\operatorname{AUTHOR}(\mathrm{S}):$}

Hasegawa, Yoshinori; Watanabe, Takashi;

Takazawa, Masaki; Ohara, Osamu; Kubota, Shin

\section{CITATION:}

Hasegawa, Yoshinori ... [et al]. De Novo Assembly of the Transcriptome of Turritopsis, a Jellyfish that Repeatedly Rejuvenates. Zoological Science 2016, 33(4): 366-371

\section{ISSUE DATE:}

2016-08

URL:

http://hdl.handle.net/2433/216317

\section{RIGHT:}

(c) 2016 Zoological Society of Japan; The full-text file will be made open to the public on 01 August 2017 in accordance with publisher's 'Terms and Conditions for Self-Archiving'. 


\title{
De Novo Assembly of the Transcriptome of Turritopsis, a Jellyfish that Repeatedly Rejuvenates
}

\author{
Author(s): Yoshinori Hasegawa, Takashi Watanabe, Masaki Takazawa, Osamu Ohara and Shin \\ Kubota
}

Source: Zoological Science, 33(4):366-371.

Published By: Zoological Society of Japan

DOI: http://dx.doi.org/10.2108/zs150186

URL: http://www.bioone.org/doi/full/10.2108/zs150186

BioOne (www.bioone.org) is a nonprofit, online aggregation of core research in the biological, ecological, and environmental sciences. BioOne provides a sustainable online platform for over 170 journals and books published by nonprofit societies, associations, museums, institutions, and presses.

Your use of this PDF, the BioOne Web site, and all posted and associated content indicates your acceptance of BioOne's Terms of Use, available at www.bioone.org/page/terms_of_use.

Usage of BioOne content is strictly limited to personal, educational, and non-commercial use. Commercial inquiries or rights and permissions requests should be directed to the individual publisher as copyright holder. 


\title{
De Novo Assembly of the Transcriptome of Turritopsis, a Jellyfish That Repeatedly Rejuvenates
}

\author{
Yoshinori Hasegawa $^{1 \star \dagger}$, Takashi Watanabe ${ }^{1}$, Masaki Takazawa ${ }^{1}$, \\ Osamu Ohara ${ }^{1}$, and Shin Kubota ${ }^{2}$
${ }^{1}$ Department of Technology Development, Kazusa DNA Research Institute, Kisarazu, Chiba 292-0818, Japan
${ }^{2}$ Seto Marine Biological Laboratory, Field Science Education and Research Center, Kyoto University, Shirahama, Wakayama 649-2211, Japan

\begin{abstract}
In most animals, aging is an irreversible process; however the species Turritopsis sp. has been observed to undergo a rejuvenation process as many as $\mathbf{1 4}$ times. In the present study, we used multiplexed RNA libraries to obtain the transcriptome from four developmental stages (St) of Turritopsis sp., including (I) immature medusa, (II) dumpling, (III) dumpling with a short stolon, and (IV) polyp, which had recently rejuvenated. A total of 4.02 billion paired-end reads were assembled de novo, yielding 90,327 contigs. Our analyses revealed that significant blast hits were recovered for $74 \%$ of the assembled contigs, and $19 \%$ were successfully annotated with gene ontology (GO) terms. A BLAST search demonstrated that $32 \%$ of the contigs were most similar to Hydra vulgaris sequences. Raw reads from each sample were mapped against the contigs to find St-specific genes. This represents the first comprehensive set of de novo transcriptome data for this species, which may provide clues toward a better understanding of cyclical rejuvenation in multicellular animals.
\end{abstract}

Key words: jellyfish, RNA-Seq, rejuvenation, de novo assembly, Turritopsis

\section{INTRODUCTION}

Life cycle reversion, which is rejuvenation from a mature or young medusa to a polyp, is a unique feature of some cnidarians, including Turritopsis spp. (Bavestrello et al., 1992; Piraino et al., 1996; Kubota, 2005), Laodicea undulata (Kubota, 2006; Vito et al., 2006), Linuche unguiculata (Vito et al., 2006) and Aurelia sp.1 (He et al., 2015). Of these, Turritopsis sp., an undescribed species (Kubota, 2009), is the only one in which repeated cycles of rejuvenation has been observed, more than 10 times in a female colony from southern Japan (Kubota, 2011). Interestingly, the same colony has continued to repeat this reversal of its life cycle, suggesting a possibility that these animals are in some sense 'immortal.' This phenomenon, however, may in fact represent a form of regeneration, since dumpling-stage animals which have degenerated from a medusa develop into polyps without passing through the planula stage, which is typical of cnidarian juveniles. We have observed that two different states of dumplings punctured with a pair of stainless needles transformed into two different forms; one developed directly into a medusa and the other into a stolon (Kubota, 2016). We believe that the latter phenomenon is a form of rejuvenation, or "life-cycle reversal," while the former

\footnotetext{
${ }^{*}$ Corresponding author. Tel. : : +81-438-52-3915;

Fax : +81-438-52-3914;

E-mail: yhasega@kazusa.or.jp

${ }^{\dagger}$ Present address. Department of Research \& Development, Kazusa

DNA Research Institute, Kisarazu, Chiba 292-0818, Japan

Supplemental material for this article is available online.

doi:10.2108/zs150186
}

represents regeneration. Elucidating the mechanisms underlying rejuvenation in this species may help to clarify the processes that lead to transdifferentiation, dedifferentiation, and aging.

Recently, a number of transcriptome studies using nextgeneration sequencing (NGS) in cnidarians have been reported (Siebert et al., 2011; Kitchen et al., 2015; Sanders and Cartwright, 2015), in addition to whole-genome sequencing of Nematostella vectensis (Putnam et al., 2007), Hydra magnipapillata (Chapman et al., 2010), and Acropora digitifera (Shinzato et al., 2011). However, to our knowledge, no molecular biological studies in the Oceaniidae have been performed. Next-generation sequencing is a useful strategy for the study of gene expression in Turritopsis sp., and it enables an important step in understanding rejuvenation in this species. In the present report, we present the now publicly available sequence of a repeatedly rejuvenating jellyfish, and highlight the genes that appear to be involved in rejuvenation.

\section{MATERIALS AND METHODS}

Sample collection and preparation

A total of 11 Turritopsis sp. medusae were collected on 14-15 August 2014 in Tanabe Bay, Japan (33.68'E, 135.36'N). All of the materials were collected along the wharf using a plankton net handtowed from the bottom ( $7 \mathrm{~m}$ deep) to the surface. After collection, immature medusae were each punctured over 100 times with a pair of stainless steel needles, and transferred individually into $60 \mathrm{cc}$ polystyrene containers filled with $5 \mu \mathrm{m}$ of filtered natural seawater and placed in an incubator maintained at $25^{\circ} \mathrm{C}$. After a few days, the polyp rejuvenated, transforming from an immature medusa to a polyp through the following four stages: (I) immature medusa, (II) 
dumpling, (III) dumpling with a short stolon, in which neither the hydranth nor hydrocaulus had yet emerged from the stolon, and (IV) newly rejuvenated polyp, consisting of only a single zooid with tentacles on the extended stolon (Fig. 1).

\section{Total RNA extraction, paired-end mRNA library preparation, and sequencing}

Each sample was initially stored in RNAlater RNA Stabilization Reagent (Qiagen, Venlo, Netherlands) at $4^{\circ} \mathrm{C}$. The individuals processed on the same treatment day at the same developmental stage (St) were pooled for library construction. All libraries were constructed from 1-3 individuals (Table 1). Six libraries were prepared, consisting of one from St I, two from St II, two from St III, and one from St IV (Table 1). Within a week after RNAlater treatment, each specimen was transferred to $800 \mu \mathrm{L}$ TRIZOL Reagent (Life Technologies, Carlsbad, CA, USA). The solution was homogenized using BioMasherll (Nippi, Tokyo, Japan) and incubated at room temperature for $5 \mathrm{~min}$. After adding $200 \mu \mathrm{L}$ chloroform and centrifugation, the aqueous phase was carefully transferred to a new tube after which $5 \mu \mathrm{g}$ of glycogen (Life Technologies) was added to samples as a co-precipitant. RNA was precipitated by adding $600 \mu \mathrm{L}$ of isopropyl alcohol. The RNA pellet was washed once with $75 \%$ ethanol and then dissolved in $15 \mu \mathrm{l}$ RNase-free water and purified with the Agencourt RNAClean XP kit (Beckman Coulter). The quality and concentration of the RNA were verified with the Agilent 2100 Bioanalyzer and Qubit Fluorometer (Life Technologies), respectively. The final amount of each sample ranged from approximately 50 to $2,500 \mathrm{pg}$ RNA. A 10-pg sample of purified total RNA was reverse transcribed and amplified to approximately $5 \mathrm{ng}$ using SMARTer Ultra Low Input RNA Kit for Sequencing v3 (Clontech Laboratories, Mountain View, CA, USA) according to the manufacturer's instructions. Amplified double stranded cDNA was sheared using Covaris, and then $1 \mathrm{ng}$ of sheared CDNA was used for library preparation. Libraries were sequenced using the Illumina HiSeq 2000 (Illumina) and prepared with the KAPA Hyper Prep Kit (Kapa Biosystems) according to the manufacturer's instructions. An Illumina CBOT was used for cluster generation, and the CDNA libraries were sequenced on an Illumina HiSeq 2000 system performing 100nt-long reads.

\section{De novo transcriptome assembly and annotation}

Prior to assembly, adapter sequences were removed from raw reads, and the trimmed reads were assessed for quality using FASTX Toolkit (v0.0.13) software. Base trimming was done from the $3^{\prime}$ end of each read to remove bases with a quality below Q10 up to a minimum length of $50 \mathrm{bp}$. Reads shorter than $50 \mathrm{bp}$ and the remaining orphan reads were removed before further analysis. The combined reads of all six libraries were assembled de novo using Trinity (Grabherr et al., 2011) (release 2014-07-17) with a minimum contig length of $200 \mathrm{bp}$ and a k-mer size of 25 . Gene ontology (GO) annotations of the contigs from the Trinity assembly were conducted through a BLASTx search against the NCBI NR protein database with a cut off E-value of 1.0E-6 using Blast2GO (v2.8).

The raw data and the de novo assembly have been deposited in the DNA Data Bank of Japan (DDBJ), (accession nos. DRA004346, PRJDB4516, SAMD00045835-SAMD00045840, DRX048560-DRX048565, IAAF01000001-IAAF01090327).

\section{RNA-Seq analysis}

Each read of the stage-specific libraries was mapped to the contigs using Bowtie software (v2.0.6) with the default settings allowing for two mismatches (Langmead et al., 2009) and the resulting BAM data, which contained all transcript variants, was imported into a Strand NGS (v2.1, Agilent Technologies) for further analysis. After normalization of the trimmed mean of M-values (TMM) (Robinson and Oshlack, 2010) with the default settings, hierarchical clustering was performed on group stage-specific samples according to the gene expression patterns using the default settings. To identify the genes expressed only in specific St, TMM values for the contigs of samples from individual St (genes from St I and IV were obtained from a single library, whereas those from St II and III were identified from a total of four libraries, two from each St) were compared to the other three St samples, at which the fold-change cutoff was set at $\geq 2.0$. Subsequently, St-specific contigs were extracted, which were expressed only in one St and were reimported to Blast2GO to perform a statistical assessment of GO term enrichment compared to a reference group using a Fisher's Exact Test. The resulting data $(P<0.01)$ was then classified by GO terms, and are available at the following URL: http://www.animalgenome. org/tools/catego/.

\section{RESULTS AND DISCUSSION}

\section{Illumina sequencing and de novo transcriptome assem- bly}

To generate a broad survey of the transcripts associated with rejuvenation, a cDNA library was constructed from a very small amount of RNA extracted from small individuals at various stages (Fig. 1). Illumina sequencing of the six libraries from four stages (composed of 11 individuals) yielded 566 million paired-end 100-bp reads (Table 1). After filtering was performed to remove low-quality and orphan reads, a total of $402,312,548$ clean reads $(71.1 \%)$ were obtained from six libraries. The de novo assembly yielded 90,327 contigs with an average length of $913 \mathrm{bp}$ and a N50 of 1,464 bp (Fig. 2, Table 2). Analysis revealed that $74.2 \%$ of the contigs had significant blast hits against the NCBI nr protein database (Table 3). Importantly, although a proportion of the short contigs in no blast hit increased, i.e., in the $201-300$ bp range (11,268 of 24,076 contigs), the substantial long-read contigs remained (average $419 \mathrm{bp}$, range 201-4440 bp). Of the contigs with hits, the species that was most often a match was Hydra vulgaris $(21,407$ hits), followed by Nematostella vectensis (4,007 hits), which belong to the same phylum as Turritopsis sp. in Cnidaria (Fig. 3). The hits for these two species represented $38.0 \%$ of the
Table 1. Sample information and sequencing yield.

\begin{tabular}{lcccr}
\hline \multicolumn{1}{c}{ Sample } & $\begin{array}{c}\text { Rejuvenation } \\
\text { Stage }\end{array}$ & $\begin{array}{c}\text { Sample } \\
\text { ID }\end{array}$ & $\begin{array}{c}\text { No. of } \\
\text { individuals used }\end{array}$ & Raw Reads \\
\hline Immature medusa & I & 1 & 1 & $85,275,248$ \\
Dumpling & II & 2 & 1 & $100,534,214$ \\
Dumpling & II & 3 & 3 & $83,660,368$ \\
Dumpling with a short stolon & III & 4 & 1 & $96,403,688$ \\
Dumpling with a short stolon & III & 5 & 3 & $106,513,140$ \\
Polyp & IV & 6 & 2 & $93,627,712$ \\
\hline
\end{tabular}

Table 2. Assembly statistics of Illumina pairedend data.

\begin{tabular}{lc}
\hline Total length (bp) & $82,460,655$ \\
Number of contigs & 90,327 \\
Average length (bp) & 913 \\
Median length (bp) & 533 \\
Max length (bp) & 85,699 \\
Min length (bp) & 201 \\
N50 (bp) & 1,464 \\
GC content & $38.67 \%$ \\
\hline
\end{tabular}




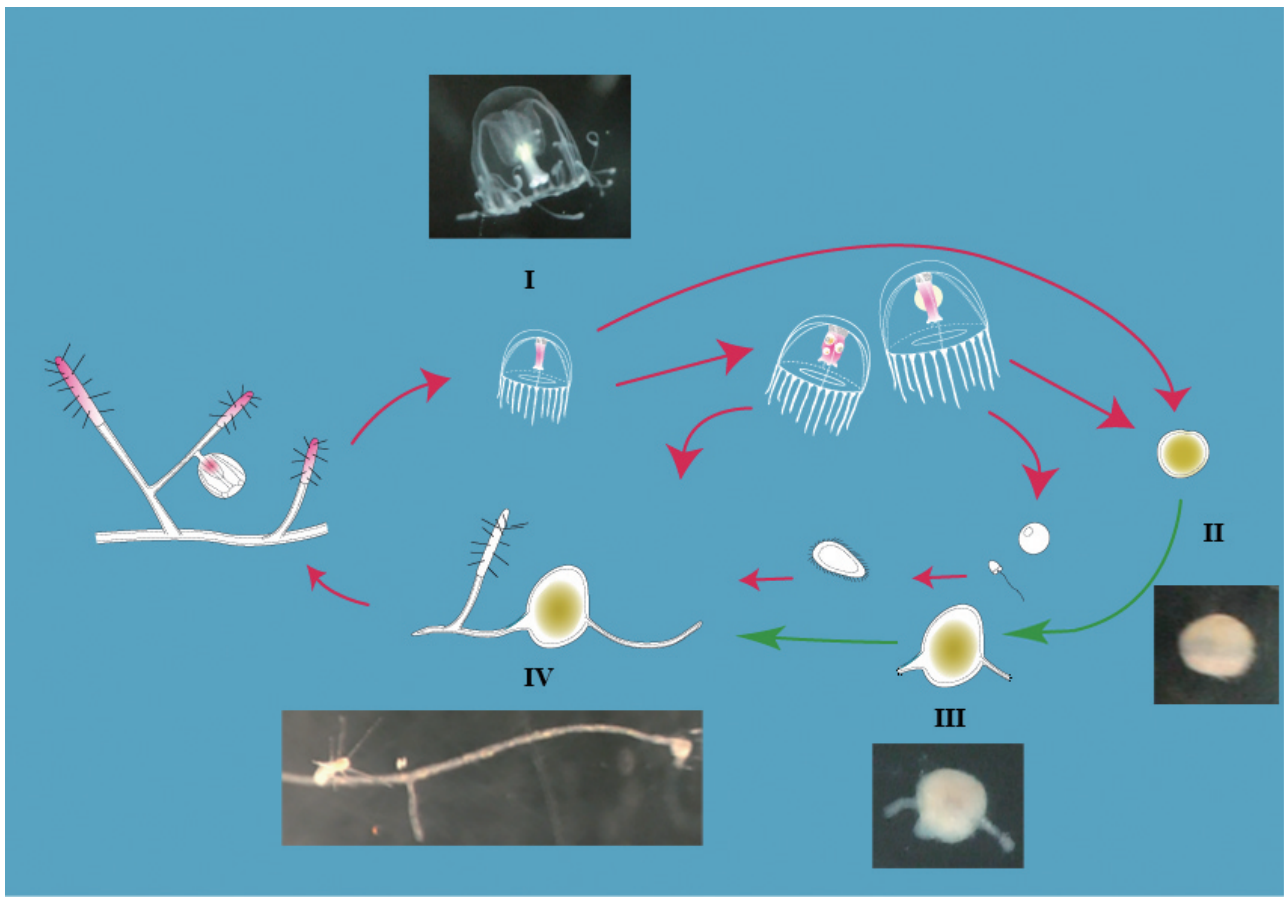

Fig. 1. Life cycle reversion showing rejuvenation from mature or young medusa to a polyp in Turritopsis sp. Developmental stages analyzed include the (I) immature medusa; (II) dumpling; (III) dumpling with a short stolon; and (IV) polyp (newly rejuvenated).

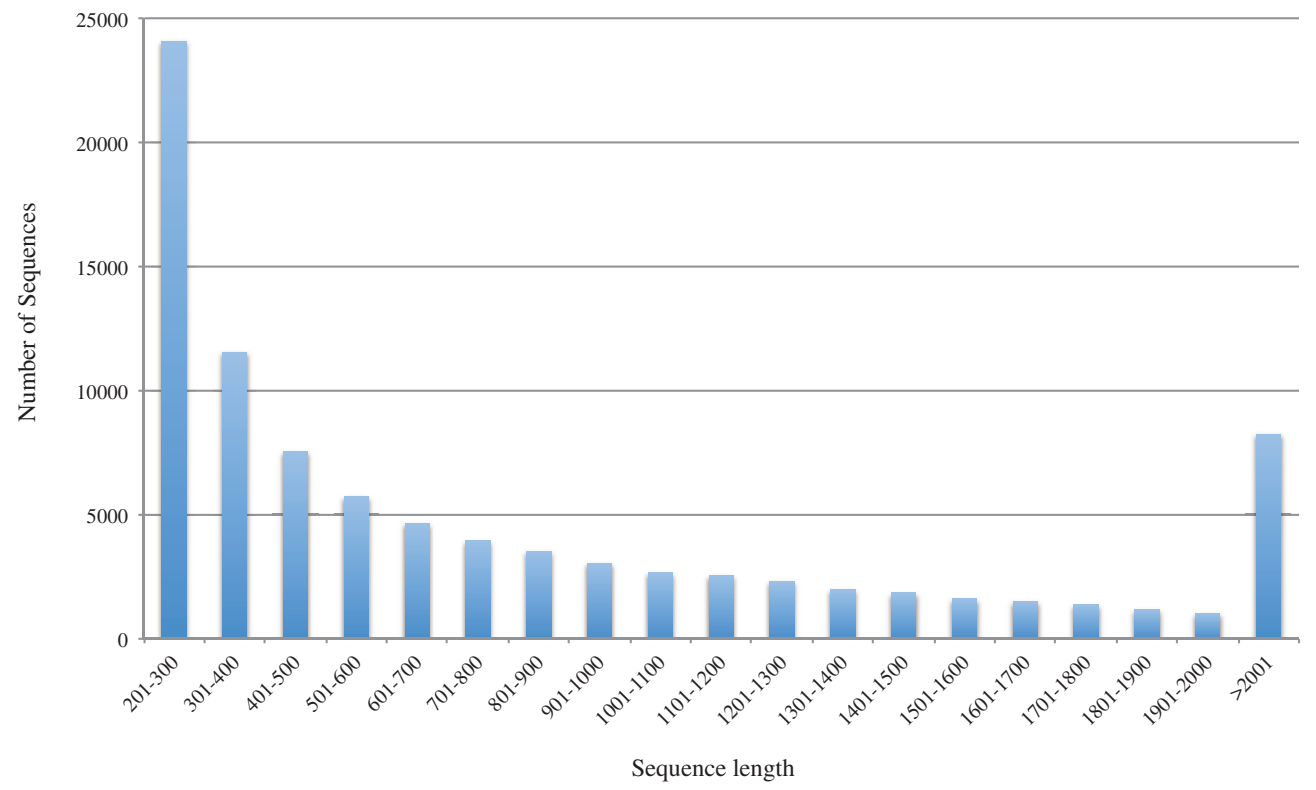

Fig. 2. Distribution of the size of assembled transcripts from Turritopsis sp. transcriptome libraries.

total hits, suggesting the high quality of the assembled genes and, notably, their conservation among Cnidaria.

\section{Expression level analysis}

In order to visualize the expression patterns of transcripts at the different stages, we conducted a hierarchical clustering analysis (Fig. 4). The expression profile across all transcripts demonstrated that it was largely divided between the medusa (St I) and all other stages (St II-St IV), and secondly revealed that the polyp (St IV) differed from the dump- lings (St II) and dumplings with short stolons (St III).

One of the most important findings of this transcriptome sequencing project was the identification of Stspecific genes from the four life stages (Supplementary Tables S1-S4 online). We found that immature jellyfish (St I) expressed the highest number of St-specific genes, followed by dumpling (St II), polyp (St IV), and dumpling with short stolon (St III) (Fig. 5). A detailed analysis of Stspecific expression states revealed that the majority of the genes in St I was expressed over $10 \times$ more than in those in St II, III and IV, which were characterized by a low level of expression (Fig. 5). In particular, Wnt signaling is regulated by a conserved pathway in metazoans and has been reported to play an important role in development (Sanders and Cartwright, 2015), the regeneration process (Chera et al., 2009; Petersen et al., 2015), and axial patterning (Petersen and Reddien, 2009) in cnidarians. There is an example of St-specific expression linked to the Wnt pathway, which is expressed mainly found in St I. Specifically, Wnt3, which is secreted by apoptotic cells at the head regeneration site and induces regeneration after injury in Hydra (Chera et al., 2009), is expressed during St I and IV, but not St II or III. The expression values after TMM normalization of for Wnt3 from contigs c74698_g1_i1 and c77967_g1_i1 from St I were 153.8 and 165.9, respectively, and that of c63687_g1_i1 from St IV was 98.6. These results suggest that although the Wnt signaling pathway is not involved at the dumpling stage, it could play an essential role after the medusa is injured (for example, after being wounded with a needle). It is also possible that this pathway is involved in the wound healing response to mechanical disruption; therefore, we are currently planning to obtain additional St samples for further analyses of transcription level changes in the life stages of Turritopsis sp. 
Table 3. Summary of de novo reference transcriptome annotation statistics using the blastx algorithm with Blast2GO software.

\begin{tabular}{ll}
\hline Total number of transcripts & 90,327 \\
Successful sequences after BLAST step & 66,984 \\
Successful sequences after Mapping step & 44,022 \\
Successful sequences after Annotation step & 17,488 \\
\hline
\end{tabular}

Mapping step is the process of retrieving GO terms associated to the hits obtained after a BLAST search.

Annotation step is the process of selecting GO terms from the GO pool obtained by the Mapping step and assigning them to the query sequences (a cut off E-value of 1.0E-6).
GO term enrichment revealed the St-specific functionalrelated gene groups (Fig. 6). St IV harbored the smallest number of enriched pathways in all three of the domains identified, most notably those in the cellular component group. Two GO terms ("cell communication" and "signal transduction") in the biological processes domain were relatively similar in abundance in all stages other than St II. However, four GO terms, "catabolism" and "secondary metabolism" in the biological process domain, along with "catalytic activity" and "DNA binding" in the molecular function domain, were found in relatively similar levels of abun-

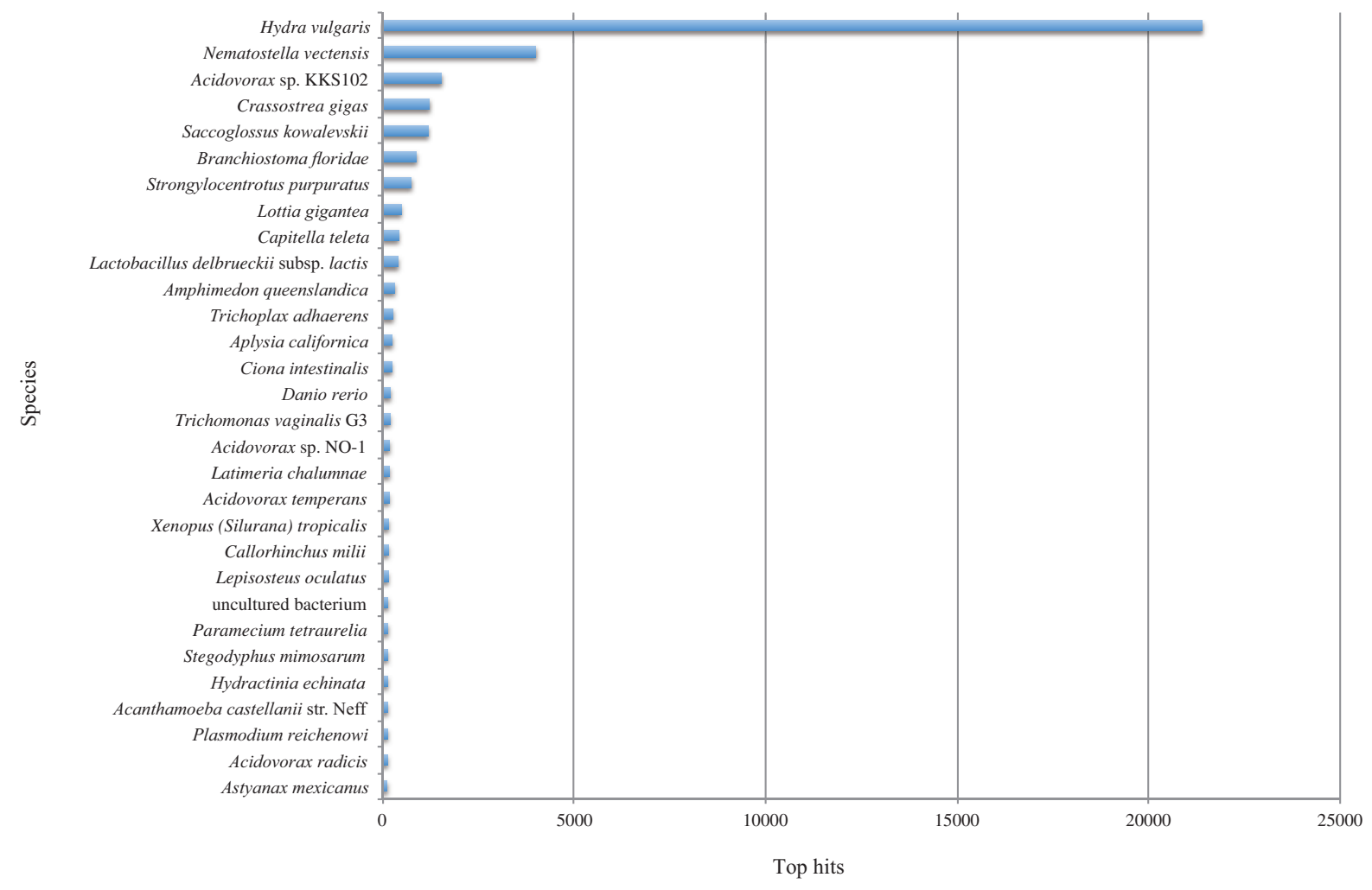

Fig. 3. Species distribution of the top hits identified by Blast2GO annotation using Turritopsis sp. sequences.

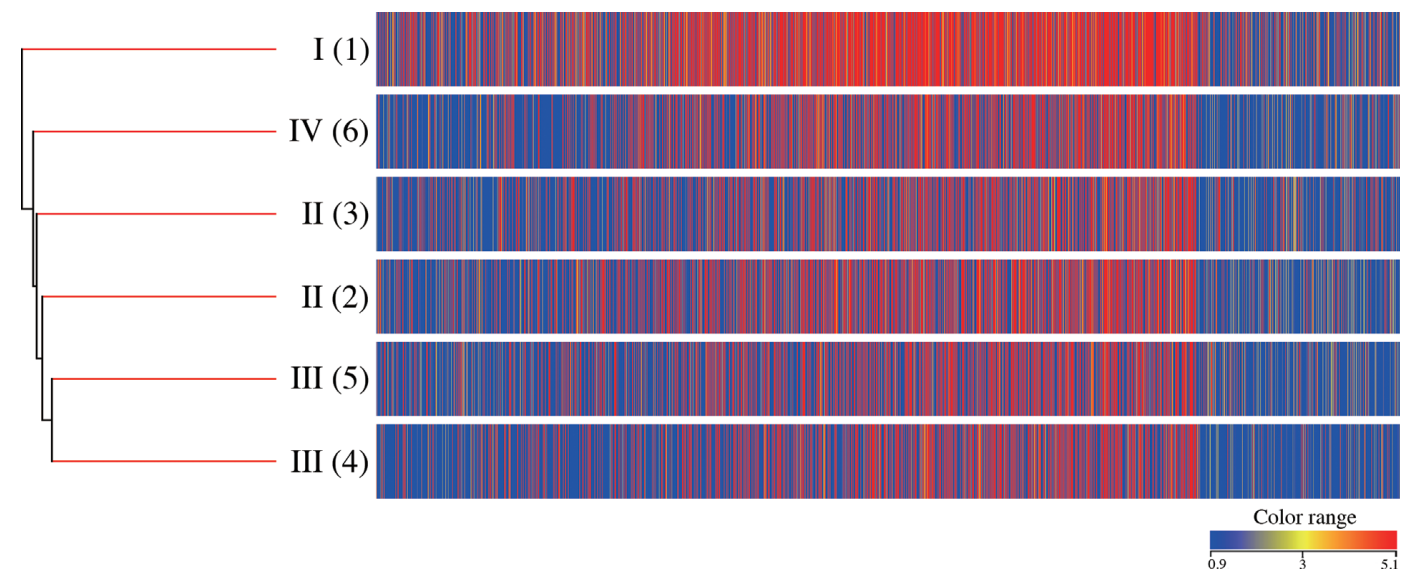

Fig. 4. Heat map comparing expression levels among all transcript libraries of Turritopsis $\mathrm{sp}$. in the four developmental stages analyzed. I, II, III, and IV indicate the developmental stages whereas the number in parenthesis is the sample ID shown in Table 1. Similarity between developmental stages with hierarchical clustering is shown above the heat map. The analysis was performed using Strand NGS, with the Hierarchical Clustering algorithm, Euclidean distance metric, and Ward's linkage rule. 




Fig. 5. Relative expression of developmental stage-specific expression for various Turritopsis sp. contigs. Relative levels of expression in comparison to the three other stages are identified as follows: the blue bar indicates 2-5 times, red indicates 5-10 times, and green indicates a minimum of 10 times greater than that in the other three stages. dance only in St II. These results may aid in the identification of genes that play essential roles in rejuvenation.

\section{Conclusion}

In this study, we identified genes and pathways expressed in specific developmental stages of Turritopsis sp., and were thus able to provide an inventory of the genes that may be involved in the rejuvenation process. One-third of the contigs from Turritopsis sp. harbored blast hits to Hydra vulgaris. Hydra is considered another example of immortality due to its constant mortality and fertility over age (Schaible et al., 2015). Turritopsis sp. and Hydra could possibly share multiple pathways, although significant differences among them may allow for a unique function in Turritopsis sp. In order to elucidate the mechanism of the initial reaction to the rejuvenation phenomenon, it is effective to investigate the combination of St II-specific expression and/or silent genes using the same method as that previously implemented to identify iPS factors (Takahashi and Yamanaka, 2006). It is also important to determine whether rejuvenation occurs only in specialized cell types, such as stem cells. While here we identified candidate genes and pathways related to rejuvenation, we suggest that it is pos-
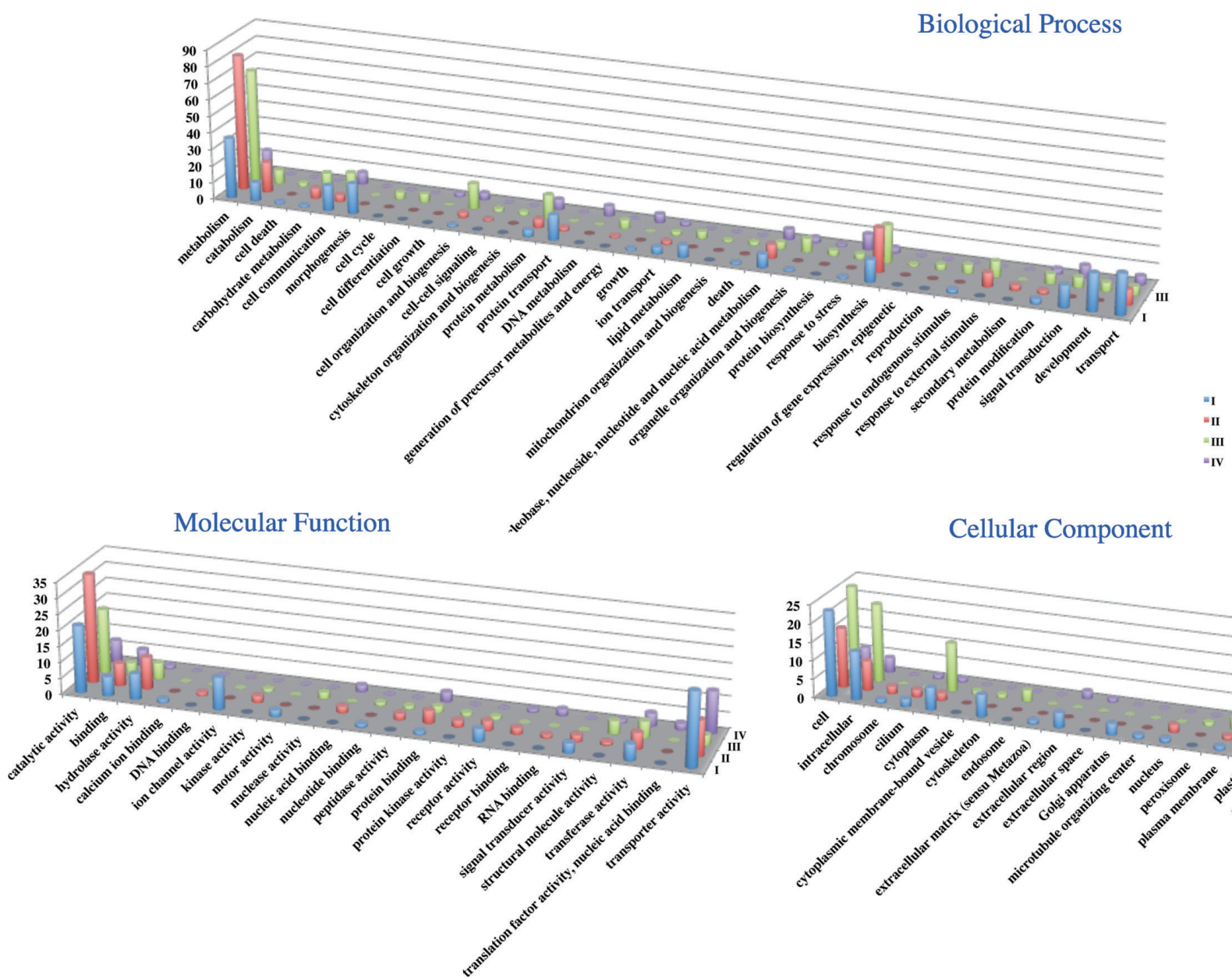

Cellular Component

Fig. 6. Comparison of the level 2 gene ontology (GO) term enrichment analysis among the developmental stage-specific gene expression in Turritopsis sp. ( $P<0.01$, Fisher's Exact Test). 
sible that as many as one-fourth of the contigs with no blast hit also play major roles in this process. In fact, analysis of 90,327 Trinity contigs using ESTscan (Iseli et al., C, 1999) produced 64,191 ORFs, and one contig was assigned an ORF for the first time. Moreover, of the 23,343 contigs with no blast hit, 98 harbored extended lengths of over $100 \mathrm{bp}$ (data not shown). We are currently working towards obtaining a whole-genome sequence for this Turritopsis sp., the immortal jellyfish. The transcriptomes presented here will enhance attempts at fully assembling the genome, and allow for the elucidation of the molecular mechanisms underlying immortality and repeated rejuvenation in Turritopsis sp.

\section{REFERENCES}

Bavestrello G, Sommer C, Sarà M (1992) Bi-directional conversion in Turritopsis nutricula (Hydrozoa). Sci Mar 56: 137-140

Chapman JA, Kirkness EF, Simakov O, Hampson SE, Mitros T, Weinmaier T, et al. (2010) The dynamic genome of Hydra. Nature 464: 592-596

Chera S, Ghila L, Dobretz K, Wenger Y, Bauer C, Buzgariu W, et al. (2009) Apoptotic cells provide an unexpected source of Wnt3 signaling to drive Hydra head regeneration. Dev Cell 17: 279289

Grabherr MG, Haas BJ, Yassour M, Levin JZ, Thomson DA, Amit I, et al. (2011) Full-length transcriptome assembly from RNA-Seq data without a reference genome. Nat Biotechnol 29: 644-652

He J, Zheng L, Zhang W, Lin Y (2015) Life Cycle Reversal in Aurelia sp. 1 (Cnidaria, Scyphozoa). PLoS One 10: e0145314

Iseli C, Jongeneel CV, Bucher P (1999) ESTScan: a program for detecting, evaluating, and reconstructing potential coding regions in EST sequences. Proc Int Conf Intell Syst Mol Biol: 138-148

Kitchen SA, Crowder CM, Poole AZ, Weis VM, Meyer E (2015) De novo assembly and characterization of four Anthozoan (phylum Cnidaria) transcriptomes. G3 (Bethesda) 5: 2441-2452

Kubota S (2005) Distinction of two morphotypes of Turritopsis nutricula medusae (Cnidaria, Hydrozoa, Anthomedusae) in Japan, with reference to their different abilities to revert to the hydroid stage and their distinct geographical distributions. Biogeography 7: 41-50

Kubota S (2006) Life cycle reversion of Laodicea undulata (Hydrozoa, Leptomedusae) from Japan. Bull Biogeogr Soc Japan 61: 8588 (in Japanese with English abstract)

Kubota S (2009) Turritopsis sp. (Hydrozoa, Anthomedusae) rejuvenated four times. Bull biogeogr Soc Japan 64: 97-99 (in Japanese with English abstract)

Kubota S (2011) Repeating rejuvenation in Turritopsis, an immortal hydrozoan (Cnidaria, Hydrozoa). Biogeography 13: 101-103

Kubota S (2016) Coming caution in physical induction of rejuvenation experiments using Turritopsis sp. (Hydrozoa, Anthomedusae) by the general run of people. Kuroshio Biosphere 12: 5357 (in Japanese with English abstract)

Langmead B, Trapnell C, Pop M, Salzberg SL (2009) Ultrafast and memory efficient alignment of short DNA sequences to the human genome. Genome Biol 10: R25

Petersen CP, Reddien PW (2009) Wnt signaling and the polarity of the primary body axis. Cell 139: 1056-1068

Petersen HO, Höger SK, Looso M, Lengfeld T, Kuhn A, Warnken U, et al. (2015) A comprehensive transcriptomic and proteomic analysis of Hydra head regeneration. Mol Biol Evol 32: 19281947

Piraino S, Boero F, Aeschbach B, Schmid V (1996) Reversing the life cycle: medusae transforming into polyps and cell transdifferentiation in Turritopsis nutricula (Cnidaria, Hydrozoa). Biol Bull 190: 302-312

Putnam NH, Srivastava M, Hellsten U, Dirks B, Chapman J, Salamov A, et al. (2007) Sea anemone genome reveals ancestral eumetazoan gene repertoire and genomic organization. Science 317: 86-94

Robinson MD, Oshlack A (2010) A scaling normalization method for differential expression analysis of RNA-seq data. Genome Biol 11: R25

Sanders SM, Cartwright P (2015) Interspecific differential expression analysis of RNA-Seq data yields insight into life cycle variation in hydractiniid hydrozoans. Genome Biol Evol 7: 24172431

Schaible R, Scheuerlein A, Dańko MJ, Gampe J, Martínez DE, Vaupel JW (2015) Constant mortality and fertility over age in Hydra. Proc Natl Acad Sci U S A 112: 15701-15706

Shinzato C, Shoguchi E, Kawashima T, Hamada M, Hisata K, Tanaka M, et al. (2011) Using the Acropora digitifera genome to understand coral responses to environmental change. Nature 476: 320-323

Siebert S, Robinson MD, Tintori SC, Goetz F, Helm RR, Smith SA, et al. (2011) Differential gene expression in the siphonophore Nanomia bijuga (Cnidaria) assessed with multiple next-generation sequencing workflows. PLoS One 6: e22953

Takahashi K, Yamanaka S (2006) Induction of pluripotent stem cells from mouse embryonic and adult fibroblast cultures by defined factors. Cell 126: 663-676

Vito DDe, Piraino S, Schmich J, Bouillon J, Boero F (2006) Evidence of reverse development in Leptomedusae (Cnidaria, Hydrozoa): the case of Laodicea undulata (Forbes and Goodsir, 1851). Mar Biol 149: 339-346

(Received November 17, 2015 / Accepted April 6, 2016) 